\title{
Investments in surgical systems contribute to pandemic readiness and health system resilience
}

\author{
Megan E. Bouchard ${ }^{1,2} \cdot$ Natalie Sheneman'2 $\cdot$ Matthew T. Hey ${ }^{2,3}$. \\ Laura Hoemeke $^{2,4} \cdot$ Fizan Abdullah ${ }^{1,2}$
}

Accepted: 24 May 2021 / Published online: 30 June 2021

(c) The Author(s), under exclusive licence to Springer Nature Limited 2021

\begin{abstract}
Safe surgical care, including anesthesia, obstetrics, and trauma, is an essential component of a functional health system, yet five billion people lack access to highquality, timely and affordable surgical care. As health decision makers are grappling with how to make appropriate investments for crisis readiness and resilience, investments in surgical care should be considered for their compounding benefits to meet a country's diverse health goals. National Surgical, Obstetric, and Anesthesia Plans (NSOAPs) are developed through global partnerships and multi-stakeholder consensus and provide a dynamic framework for surgical scale-up that also improves the resilience of the larger health system. Our paper applies principles from the literature on health system resilience to surgical systems and examines the unique capabilities of the surgical workforce and infrastructure to be redeployed during times of crisis, using examples from the current pandemic.
\end{abstract}

Keywords Health system resilience $\cdot$ Pandemic readiness $\cdot$ Surgical investment

\section{Key messages}

- Investments in a country's surgical infrastructure can not only improve access and the quality of surgical care but also the resilience of the larger health system.

Fizan Abdullah

fabdullah@1uriechildrens.org

1 Division of Pediatric Surgery, Department of Surgery, Ann \& Robert H. Lurie Children's Hospital of Chicago, Northwestern University Feinberg School of Medicine, 225 E. Chicago Ave, Chicago, IL 60611, USA

2 The G4 Alliance, Chicago, IL, USA

3 Herbert Wertheim College of Medicine, Florida International University, Miami, FL, USA

4 Gillings School of Global Public Health, University of North Carolina-Chapel Hill, Chapel Hill, NC, USA 
- Strengthening surgical capacity in LMICs, particularly through well-developed and financed NSOAPs, bridges gaps in surgical care while simultaneously offering adaptable infrastructure, equipment, and a skilled specialist workforce to respond to other health crises.

\section{Background}

Safe surgical care, including anesthesia, obstetrics, and trauma, is an essential component of a functional health system, yet five billion people lack access to high-quality, timely, and affordable surgical care [1,2]. With surgically treatable conditions comprising one third of the global burden of disease, investment in surgical systems strengthening is urgently needed to reduce preventable deaths, disabling morbidity, and loss of economic productivity [1,3]. Because member states of the World Health Organization (WHO) committed to the inclusion of emergency and essential surgical care in the WHO definition of universal health coverage (UHC) in 2015, many low- and low middle-income countries (LMICs) have developed National Surgical, Obstetric, and Anesthesia Plans (NSOAPs) to engage critical stakeholders in the strategic allocation of resources and to identify opportunities to improve surgical access within their respective countries [4]. NSOAPs can serve as a dynamic framework to improve the stability of a health system overall [4]. Indeed, many of the necessary components of a strong surgical system parallel the needs required for a timely, robust response to other unexpected health threats, including pandemics. Surgical systems strengthening necessitates increasing the specialist workforce, supply chain, critical infrastructure, and existing triage systems. These systems are well positioned to reinvent themselves for other uses. As countries work to strengthen their health systems for crisis readiness and resilience, investments for surgery are likely to have compounding benefits to meet countries' other health goals.

\section{Resilience as a component of health system strengthening}

The concept of resilience is often referenced in context to health systems strengthening [5-7]. Resilience addresses the ability of a national health system to react and adapt under changing circumstances [8]. These include not only 'shocks' like pandemics, natural disasters, and political upheaval but also gradual shifts in epidemiological, socioeconomic, and environmental factors that affect health. While there are various definitions and theories of resilience [5, 7-10], these interpretations share an understanding that a health system's resilience is the responsibility of national governments and it relates to a combination of multisectoral institutions, societal preconditions, and adaptable capacity designed for both current and potential needs within a specific country. Resilient health systems also share certain traits. Pulling from multidisciplinary literature on resilience, Kruk et al. characterized resilient health systems as integrated, diverse, aware, self-regulated, and adaptive [8].

While health system resilience is infrequently discussed outside of health crises, it has implications for both routine care and unexpected health threats. A 
prerequisite of a health system's resilience is its ability to provide comprehensive care during 'normal times' when there is no perceived crisis [7]. The 2014 Ebola outbreaks in Guinea, Liberia, and Sierra Leone resulted in disproportionately high health and economic impacts, largely because these countries had gaps in routine care and lacked resilience mechanisms, such as surveillance and strong information and communication systems [11]. In the first few days following the 2015 earthquake in Nepal, patients requiring surgery were entirely reliant on the limited capacity and services of a single local hospital, resulting in calls for integrated disaster preparedness and surgical development frameworks [12]. Similarly, the impact of the ongoing COVID-19 pandemic on health systems in low- and middle-income countries (LMICs) halts progress on strengthening surgical systems in countries that were previously struggling to provide surgical care for everyone who needed it. Unlike high-income countries (HICs) where capacity is high and elective surgeries comprise a large proportion of total surgical volume, many LMICs lack the flexible hospital 'surge' capacity for pandemic response made available by canceling nonemergent surgery [13].

\section{Surgical systems planning as a framework for resilience implementation}

Theory on resilience provides limited guidance on its implementation. A 2019 literature review found an abundance of "high-level attributes" but no identifiable implementation framework for health systems resilience [14]. There is clearly a need for specific direction on the infrastructure and capabilities necessary for health systems that are resilient in both every day and unexpected care situations. A surgical systems development framework may be useful in working to strengthen a health system and build resilience. As described by the Lancet Commission on Global Surgery in 2015, surgery is a "treatment modality" rather than a "discrete disease category" [1]. Due to their complex and multidisciplinary needs, surgical systems overlap with nearly all specialties and an array of medical conditions; thus, their development supports the development of the health system overall (Fig. 1). For example, strong surgical systems support the triage, referral, and health service delivery for non-surgical care including obstetrics, oncology, trauma, critical care, and many more specialties. Furthermore, comprehensive surgical systems display many of the attributes of resilience. Applying Kruk's characteristics of resilience, they are vertically and horizontally integrated across medical issues, health system tiers, and sectors of the economy; they address a diverse range of health issues and involve a diverse workforce of providers and technicians; they require a high-level awareness of capacity to address shifting patient and population needs; they have built-in mechanisms for self-regulation, such as for referrals or transfers for specialty care; and they are adaptive to both surgical and emergent, non-surgical services.

An NSOAP, one of the most widely used surgical system implementation frameworks, provides a detailed road map for designing and executing many of the system components that foster resilience. It is a dynamic, strategic plan for the processes, timeline, budget, and metrics involved in surgical scale-up [15]. The development 


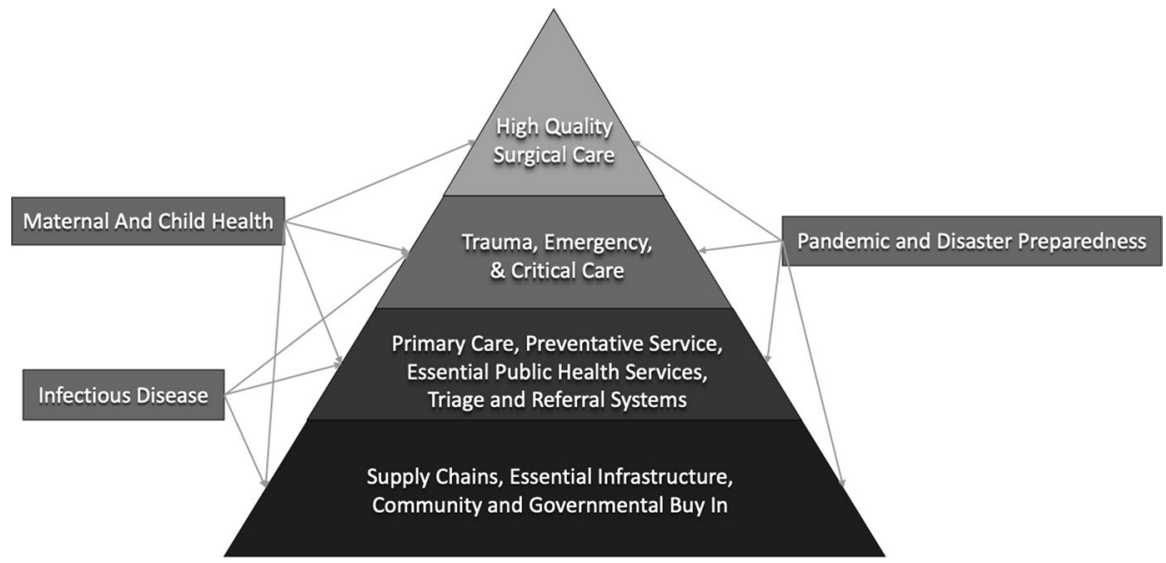

Fig. 1 Surgical systems that are able to deliver safe, affordable, and timely surgical, obstetric, and anesthesia care rely on foundational support from components of a comprehensive health care system that provides crucial, non-surgical care. Thus, a strong surgical system is often an indicator of a strong overall health system

of an NSOAP also involves consensus-building among stakeholders at the local, regional, and national levels to prioritize and integrate surgical, obstetric, and anesthesia care within national health plans and the country context [4]. These stakeholders are responsible for technical and financial inputs, as well as the implementation, evaluation, and refinement of the strategy [15]. Not unique to NSOAP development, however, whenever there is an ambitious agenda and limited resources, the political context can complicate discussions on governance and prioritization of goals. Nevertheless, the NSOAP is a useful tool for involving all critical stakeholders in developing consensus, to evaluate the effectiveness of implementation, and to exploit opportunities to meet both the needs of surgical systems and of other overlapping health priorities [15]. Ultimately, the complexity and needs of surgical systems necessitate explicit implementation plans for infrastructure, workforce, service delivery, financing, and information systems. Given their resilient characteristics and broad foundation of health system components, surgical systems can, thus, serve as a representative measure of overall health system functional and adaptable capacity.

\section{Resilient surgical system components adapted for pandemic readiness}

A surgical system that provides safe, timely, and affordable care necessitates an integrated foundation of infrastructure, supply chains, and providers beyond just the surgical team. For example, personal protective equipment (PPE) and sterilization techniques used in surgery are also necessary for providing care to patients on a medical ward and for sterilizing equipment needed to perform bedside procedures or intubate a critical patient in the intensive care unit (ICU). Oxygen and ventilators are crucial not only in providing anesthesia care but also in caring for patients with 
infectious diseases, heart failure, and chronic lung disease. Anesthesia ventilators have been adapted for COVID-19 treatment [16]. The medications required for safe anesthesia are also used for patients critically ill from other medical causes. Surgical care requires multidisciplinary expertise, including skilled nursing, critical care specialists, biomedical engineers, rehabilitation therapists, and more, all of whom are vital in other aspects of medical care too. The care of trauma patients, often led by surgeons or emergency specialists, requires robust triage and timely mobilization of resources for acute injuries; likewise, the trauma workforce and infrastructure are well positioned to respond to other health crises. Many surgical patients also require elective referrals or emergent transfers to higher-level centers of care, relying on a strong network of hospital triage and communication platforms that are beneficial for other medical issues too. Although there are many other examples, it is clear that targeted investment in surgical system strengthening provides dividends for an entire health system.

The Lancet Commission on Global Surgery highlighted the dire need to increase the global surgical workforce, especially in LMICs where many patients experience long waits and face catastrophic expenses to undergo surgery [1]. With 28.4 million non-emergent surgical operations postponed due to COVID-19 (during the initial 12 weeks of the pandemic starting in 2019) [17], surgeons, anesthetists, nurses, biomedical technicians, and other operating room staff in HICs were able to be remobilized to assist in the triage and care of COVID-19 patients. For example, the Department of Surgery at the University of Wisconsin School of Medicine restructured its entire surgical service into small teams that could easily serve alongside a critical care physician [18]. The adaptability of surgical providers, seen around the world, displays the diversity in care this highly trained workforce can provide, both in tandem with critical care physicians and independently [19, 20]. Anesthesiologists also have unmatched experience in the effective intubation of patients, a procedure that places providers at the highest risk in an aerosolized infectious disease, and extensive training in critical care $[18,21]$. For these providers to be redistributed in response to emerging health threats, however, they must already be integrated into the health system. NSOAPs address the necessary investments in training and retention of surgical providers and, thus, should increase the surgical workforce to provide not only routine surgical care, but also to respond to other crises as needed.

It is well known that many LMICs lack sufficient ICU bed capacity [22], but even in countries with a high volume of ICU beds, many hospitals have reached full capacity during the COVID-19 pandemic and have had to develop creative solutions to meet the demand for care. For example, in just three days, some hospitals increased their ICU bed capacity by $52 \%$ through retroverting existing operating rooms (ORs) or post-anesthesia care units (PACUs) into additional critical care beds [23]. However, some ORs lacked the appropriate infrastructure, such as water lines or piped oxygen, to allow conversion to ICU beds, highlighting the importance of designing flexible infrastructure [23]. Many LMICs experience gaps in critical care due to the high maintenance costs associated with ventilators [24]. Newly established guidelines to use anesthesia carts as long-term ventilators allows a more affordable solution for health systems to efficiently expand their critical care capacity [16]. By incorporating the infrastructure and equipment needed for surgical care 
with adaptation plans in the event of emerging health threats, NSOAPs present a strong investment case for overall health system strengthening for both surgical and non-surgical care.

\section{Surgical system compatibility with public health}

Even the strongest surgical systems do not replace the need for public health intervention and surveillance strategies. True pandemic and disaster preparedness incorporates preventative and reactionary capacity that work harmoniously. Where many LMICs have excelled to-date in limiting transmission of the coronavirus [25, 26], some HICs have been unable to control the pandemic due to inadequate surveillance, poor community 'buy-in,' or fragmented governance. At the same time, HICs with robust surgical and health infrastructure have managed to care for an onslaught of critically ill patients, yet many LMICs are not yet capable of an effective response to this vigorous surge capacity. With the uncertainty of future pandemics and the evolution of emerging pathogens, no one can guarantee that a strong public health surveillance system will be enough. Also, one successful country's approach is not necessarily applicable to all countries. The NSOAP, as a dynamic - and countryspecific_-strategic framework to strengthen a country's surgical system, can be a tool for developing context-specific response plans to future pandemics and other emerging health threats. Strengthening surgical capacity in LMICs, particularly through well-developed and financed NSOAPs, bridges gaps in surgical care while simultaneously offering adaptable infrastructure, equipment, and a skilled specialist workforce to respond to other health crises.

\section{Conclusion}

Investment in surgical system strengthening can lead to increased resilience of the health system overall. The essential elements include enhanced capacity to adaptively respond to emerging crises, improved global economic productivity with decreased morbidity and mortality from surgically treatable diseases, and increased care for the five billion people lacking surgical care worldwide [1]. A surgical systems framework, such as NSOAPs, can serve as a tool to improve a health system's functionality in both routine and crisis care. Investment in surgical system strengthening is uniquely tied to pandemic and other health threat responses, as surgical systems effectively serve as resilient capacity.

In the midst of a global pandemic, it is important to recognize that health systems are interconnected and that the strength of one country's health system impacts the health of other countries around the world. Therefore, the need for resilient health systems globally is apparent, urgent, and also necessary. Global surgery can serve as a pillar of pandemic preparedness and empower both LMIC and HICs to quickly adapt and respond to emerging health crises. 
Funding No funding was secured for this study. Assistance with the study: The authors formed the U.S. Resource Mobilization Working Groupof The G4 Alliance, which supported the development of this paper.

\section{Declarations}

Conflict of interest The authors have no conflicts of interest relevant to this article to disclose.

\section{References}

1. Meara JG, Leather AJ, Hagander L, et al. Global surgery 2030: evidence and solutions for achieving health, welfare, and economic development. Lancet. 2015;386(9993):569-624. https://doi.org/10. 1016/S0140-6736(15)60160-X.

2. Price R, Makasa E, Hollands M. World health assembly resolution wha68. 15: "strengthening emergency and essential surgical care and anesthesia as a component of universal health coverage"addressing the public health gaps arising from lack of safe, affordable and accessible surgical and anesthetic services. World J Surg. 2015;39:2115-25.

3. Shrime MG, Bickler SW, Alkire BC, Mock C. Global burden of surgical disease: an estimation from the provider perspective. Lancet Glob Health. 2015;3(Suppl 2):S8-9. https://doi.org/10.1016/ S2214-109X(14)70384-5.

4. Truché P, Shoman H, Reddy CL, et al. Globalization of national surgical, obstetric and anesthesia plans: the critical link between health policy and action in global surgery. Global Health. 2020;16(1):1.

5. Topp SM. Power and politics: the case for linking resilience to health system governance. BMJ Glob Health. 2020;5(6):e002891.

6. Olu O. Resilient health system as conceptual framework for strengthening public health disaster risk management: an African viewpoint. Front Public Health. 2017;5:263.

7. Kruk ME, Ling EJ, Bitton A, et al. Building resilient health systems: a proposal for a resilience index. BMJ. 2017. https://doi.org/10.1136/bmj.j2323.

8. Kruk ME, Myers M, Varpilah ST, Dahn BT. What is a resilient health system? Lessons from Ebola. Lancet. 2015. https://doi.org/10.1016/S0140-6736(15)60755-3.

9. Rodin J. The resilience dividend: being strong in a world where things go wrong. 1st ed. New York: Public Affairs; 2014.

10. Kutzina J, Sparkesa SP. Health systems strengthening, universal health coverage, health security and resilience. Bull World Health Organ. 2016;94(1):2.

11. Kieny MP, Dovlo D. Beyond Ebola: a new agenda for resilient health systems. Lancet. 2015;385(9963):91-2.

12. Pyda J, Patterson RH, Caddell L, Wurdeman T, Koch R, Polatty D, et al. Towards resilient health systems: opportunities to align surgical and disaster planning. BMJ Glob Health. 2019;4(3):e001493.

13. Mazingi D, Navarro S, Bobel MC, Dube A, Mbanje C, Lavy C. Exploring the impact of COVID-19 on progress towards achieving global surgery goals. World J Surg. 2020;44(8):2451-7.

14. Nuzzo JB, Meyer D, Snyder M, Ravi SJ, Lapascu A, Souleles J, et al. What makes health systems resilient against infectious disease outbreaks and natural hazards? Results from a scoping review. BMC Public Health. 2019. https://doi.org/10.1186/s12889-019-7707-z.

15. Johnson W, Lin Y, Mukhopadhyay S, Meara J, editors. Surgical care systems strengthening: developing national surgical, obstetric and anaesthesia plans. World Health Organ. 2017. https://apps. who.int/iris/bitstream/handle/10665/255566/9789241512244-eng.pdf;jsessionid=A258EB7033 E096BEF6BB434A2DF0D894?sequence=1. Accessed 17 Jul 2020.

16. APSF/ASA Guidance on Purposing Anesthesia Machines as ICU Ventilators. 2020. https://www. asahq.org/in-the-spotlight/coronavirus-covid-19-information/purposing-anesthesia-machines-forventilators. Accessed 27 Aug 2020.

17. COVIDSurg Collaborative. Elective surgery cancellations due to the COVID-19 pandemic: global predictive modelling to inform surgical recovery plans. Br J Surg. 2020. https://doi.org/10.1002/bjs. 11746. 
18. Zarzaur BL, Stahl CC, Greenberg JA, Savage SA, Minter RM. Blueprint for restructuring a Department of Surgery in concert with the health care system during a pandemic: the University of Wisconsin experience. JAMA Surg. 2020. https://doi.org/10.1001/jamasurg.2020.1386.

19. Bonalumi G, di Mauro M, Garatti A, Barili F, Gerosa G, Parolari A, Italian Society for Cardiac Surgery Task Force on COVID-19 Pandemic. The COVID-19 outbreak and its impact on hospitals in Italy: the model of cardiac surgery. Eur J Cardiothorac Surg. 2020;57:1025-8.

20. Ademuyiwa AO, Bekele A, Berhea AB, Borgstein E, Capo-Chichi N, Derbew M, et al. COVID-19 preparedness within the surgical, obstetric, and anesthetic ecosystem in sub-Saharan Africa. Ann Surg. 2020;272(1):e9-13.

21. Miller L, Luković E, Wagener G. Guiding airway management and personal protective equipment for COVID-19 intubation teams. Br J Anaesth. 2020. https://doi.org/10.1016/j.bja.2020.06.001.

22. Ma X, Vervoort D. Critical care capacity during the COVID-19 pandemic: global availability of intensive care beds. J Crit Care. 2020;58:96-7.

23. Peters AW, Chawla KS, Turnbull ZA. Transforming ORs into ICUs. N Engl J Med. 2020;382:e52.

24. Turner HC, Van Hao N, Yacoub S, Hoang VMT, Clifton DA, Thwaites GE, Dondorp AM, Thwaites CL, Chau NVV. Achieving affordable critical care in low-income and middle-income countries. BMJ Glob Health. 2019;4:e01675.

25. Ihekweazu C, Agogo E. Africa's response to COVID-19. BMC Med. 2020. https://doi.org/10.1186/ s12916-020-01622-w.

26. Fall IS, Rajatonirina S, Yahaya AA, Zabulon Y, Nsubuga P, Nanyunja M, et al. Integrated Disease Surveillance and Response (IDSR) strategy: current status, challenges and perspectives for the future in Africa. BMJ Glob Health. 2019;4:e001427.

Publisher's Note Springer Nature remains neutral with regard to jurisdictional claims in published maps and institutional affiliations.

Megan E. Bouchard, MD, is a Post-Doctoral Research Fellow at the Division of Pediatric Surgery, Northwestern University Feinberg School of Medicine, Ann \& Robert H. Lurie Children's Hospital of Chicago, Chicago, IL, USA and an Outreach Associate at the G4 Alliance, Chicago, IL, USA.

Natalie Sheneman, BA, is the Advocacy Associate at the G4 Alliance, Chicago, IL, USA.

Matthew T. Hey, BS, is an intern at the G4 Alliance, Chicago, IL, USA and a medical student at the Herbert Wertheim College of Medicine, Florida International University, 11,200 SW 8th St, Miami, Florida, USA.

Laura Hoemeke, MPH, DPH, is an Executive Board Member at the G4 Alliance, Chicago, IL, 60611, USA and a professor at Gillings School of Global Public Health, University of North Carolina-Chapel Hill, 135 Dauer Dr, Chapel Hill, NC, USA.

Fizan Abdullah , MD, PhD, is a Professor of Surgery and Head of the Division of Pediatric Surgery, Northwestern University Feinberg School of Medicine, Ann \& Robert H. Lurie Children's Hospital of Chicago, Chicago, IL, USA and Vice Chairman of the Board of Directors at the G4 Alliance, Chicago, IL, USA. 